\title{
The epistemic schism of statistical mechanics
}

\author{
(El cisma epistémico de la mecánica estadística)
}

\author{
Javier ANTA* \\ University of Barcelona - LOGOS - BIAP
}

\begin{abstract}
In this paper I will argue that the two main approaches to statistical mechanics, that of Boltzmann and Gibbs, constitute two substantially different theoretical apparatuses. Particularly, I defend that this theoretical split must be philosophically understood as a separation of epistemic functions within this physical domain: while Boltzmannians are able to generate powerful explanations of thermal phenomena from molecular dynamics, Gibbsians can statistically predict observable values in a highly effective way. Therefore, statistical mechanics is a counterexample to Hempel's (1958) symmetry thesis, where the predictive capacity of a theory is directly correlated with its explanatory potential and vice versa.
\end{abstract}

KEYWORDS: statistical mechanics; explanation; prediction; Hempel; Boltzmann; Gibbs.

RESUMEN: En este trabajo argumentaremos que los dos principales enfoques de la mecánica estadistica, el boltzmanniano y el gibbsiano, constituyen dos aparatos teóricos sustancialmente diferentes. En particular, defenderemos que esta división teórica debe entenderse filosóficamente como una separación de funciones epistémicas dentro de este dominio físico: mientras que los boltzmanianos son capaces de generar poderosas explicaciones de los fenómenos térmicos a partir de la dinámica molecular, los gibbsianos pueden predecir estadisticamente los valores observables de una manera altamente efectiva. Por tanto, la mecánica estadistica es un contraejemplo de la tesis de simetría de Hempel (1958), según la cual la capacidad de predicción de una teoría está directamente correlacionada con su potencial explicativo y viceversa.

PALABRAS CLAVE: mecánica estadística; explicación; predicción; Hempel; Boltzmann; Gibbs.

* Correspondence to: Javier Anta. Departament de Filosofia, Carrer de Montalegre 6-8, 4. ${ }^{\circ}$ floor, 0 ffice 4013 (PC: 08001 Barcelona) antajav@gmail.com - https://orcid.org/0000-0002-4409-7719

How to cite: Anta, Javier (2021). "The epistemic schism of statistical mechanics»; Theoria. An International Journal for Theory, History and Foundations of Science, 36(3), 399-419. (https://doi.org/10.1387/theoria.22134).

Received: 2020-10-16; Final version: 2021-03-08.

ISSN 0495-4548 - eISSN 2171-679X / (C) 2021 UPV/EHU

cC)(7) $\odot$ This work is licensed under a

Creative Commons Attribution-NonCommercial-NoDerivatives 4.0 International License 


\section{Introduction}

Statistical mechanics is, together with quantum mechanics and relativistic physics, one of the main disciplines of modern physics. It consists essentially in the study of the macroscopic behavior of certain systems, such as a gas expanding in a container, from the behavior of its microscopic components. However, unlike the other physical disciplines, statistical mechanics not only does not possess a single canonical theoretical apparatus, but there is no consensus within the philosophical literature about its fundamental problems (Wallace, 2020). Both the basic concepts and the technical procedures embedded in this discipline depend constitutively on the particular research program or approach adopted, mainly those known as Boltzmannian and Gibbsian. The fundamental problem lies in the fact that the theoretical elements of both formalisms are not primarily developed to satisfy the same epistemic functions.

In this paper I will argue that this fundamental difference between Boltzmannian and Gibbsian statistical mechanics should be understood not only as two different types of physical theories, as recently advocated by Frigg and Werndl (2019), but properly as two theoretical apparatuses with different epistemic capabilities. On the one hand, the Boltzmannian program was historically conceived to give an explanation of certain thermal phenomena, such as the second law of thermodynamics, from the movement of its molecular components (Uffink, 2007). Almost a century and a half later, Neo-Boltzmannians still aim to offer powerful explanations of certain macroscopic behavior from the dynamic evolution of the configuration of its components (e.g. Lebowitz, 1993; Callender, 1999; Albert, 2000). However, their ability to predict such behavior is extremely computationally-expensive due to the complexity of the statistical calculations involved. On the other hand, the Gibbsian program, undoubtedly the hegemonic formalism within actual statistical mechanical practices, symbolizes a significant progress in terms of computational tractability of the required statistical mechanical calculations, which is necessary to carry out effective predictions (Wallace, 2020). However, there is a consensus in the literature on the Gibbsian inability to explain. Thus, this 'epistemic schism' in the core of statistical mechanics would be a clear counterexample to Hempel's (1958) symmetry thesis, where the explanatory capacity of a theoretical apparatus is directly correlated to its predictive capacity, and vice versa.

The plan for this paper is the following. First, I will explore the basis of statistical mechanics, pointing out the main differences between Boltzmann's and Gibbs' approaches. In Section 3, I will detail the conception of the equilibrium state of each of these formalisms, as well as their descriptions of the equilibrium approach as a properly thermal behavior. Next, I will argue that these differences between Boltzmannians and Gibbsians should be understood not only as a fundamental separation in their theoretical apparatuses, but as a schism in the fundamental epistemic capabilities of statistical mechanics. On the one hand, I will try to show (Section 5) the potential of Boltzmann's statistical mechanics to generate powerful explanations of thermal behavior, on the other hand, I will analyze (Section 6) the capacity of Gibbs' statistical mechanics to derive effective predictions of these phenomena. Finally, I argue that this epistemic cleavage of statistical mechanics constitutes a clear counterexample to Hempel's symmetry thesis. 


\section{Framing Statistical Mechanics}

Statistical mechanics (SM) is the branch of modern physics ${ }^{1}$ that studies the macroscopic behavior of certain systems from the dynamics of the microscopic components of these systems. For this, SM descriptions are usually made from the space of possible molecular variables associated to the system, or simply 'phase space' $\Gamma$, which is a 6 -dimensional space (wherein $\mathrm{n}$ is the number of components of the system) that encodes all the possible dynamic values of position and momentum of the components of the system. For example, if the system described is a real gas in a closed container, then the phase space associated with the system will be of the order of $10^{24}$ dimensions.

\subsection{Basic Elements of Statistical Mechanical Descriptions}

In this statistical mechanical context, the unit of basic mechanical description (inherited from classical mechanics) is usually the 'microstate' of the system, in which all the position and moment values of all its components are determined at a given moment. Each individual microstate of the system is represented by a single point in the phase space $\Gamma$, determining all the microdynamic properties of the system at a time. However, effectively calculating this amount of microstatistical ${ }^{2}$ data is practically impossible with actual computational means (not to mention those that Boltzmann or Gibbs themselves had), for this reason the actual microstate of the system represented is usually considered as 'epistemically inaccessible' for the agent: "if these microstates were epistemically accessible, then probabilities would simply not have to be introduced in the theory and we would be able to use classical mechanics to describe such a system" (McCoy, 2020, p. 5).

If the dynamic evolution of the constituent elements of the system can be completely determined by the equations of motion of classical Newtonian or Hamiltonian mechanics (let us suppose that this is the case), then the evolution of the actual microstate of the system will be determined in turn by a particular equation of motion or evolution function. ${ }^{3}$ The dynamic evolution function of the microstate of the system, classically deterministic and time-invariant (that is, the same solution is compatible forward in time as it was backward), specifies the exact trajectory that the representative phase point will travel along the dynamically accessible phase space of the system. Again, it is pragmatically impossible to calculate this evolutionary function for systems with a physically significant number of components.

On the other hand, the basic thermodynamic description unit (itself inherited from phenomenological thermodynamics) in the SM domain is the 'macrovariable' or observational variable of the system, such as pressure or volume in the example of a gas in a vessel. Note that some macrovariables in thermodynamics are directly observable and others indirectly observable, such as Kelvin temperature and Clausius entropy, respectively. The pre-

1 Due to extension reasons, in this paper I will focus exclusively on classical statistical mechanics, and not on its quantum counterpart.

2 Although it might be a bit cumbersome, the term 'microstatistical' can be used as a shortcut for expressions like 'based on (or 'related to') microstates of the system'.

3 This equation of motion is the famous Boltzmann equation, which specifies deterministically the evolution of all components of a kinetic or molecular system (Uffink 2007). 
fix 'macro-' does not directly appeal to the fact that the property is macroscopic (although it usually is), but that it is a property whose value can be observationally determined. Each of the macrovariables $f$ that observationally describe the system y are represented in statistical mechanics approach by certain functions that associate a real number to each individual microstate of the system.

However, SM would be an incomplete theory just considering those elements, since it would not be possible to describe (much less explain or predict) the macroscopic behavior of systems based on the dynamics of their components. For this purpose, it would be necessary to specify a theoretical mechanism that would allow connecting the macrovariables of the system with their possible microvariables (i) skipping the substantial pragmatic inconveniences derived from the impossible access to the individual microstates and their deterministic evolution, and (ii) making the microstatistical time-reversibility derived from classical mechanics compatible with the time-irreversibility manifested by the empirical laws of thermodynamics governing the macrovariables. However, this theoretical mechanism will be significantly different depending on the particular approach considered, whether it is the Boltzmannian or the Gibbsian one (Wallace, 2020, pp. 586-588). This is mainly because, on the one hand, the probabilistic assumptions required to solve computationally the pragmatic intractability of the microstates ${ }^{4}$ in (i) will be completely different. On the other hand, the fit of the observationally significant description level of thermodynamics with the deterministic description level of classical mechanics in (ii) will not be common either, except for certain shared assumptions about the initial conditions ${ }^{5}$ (Albert, 2000). Let us see in detail what these differences consist of.

\subsection{Core Theoretical Elements in Boltzmann and Gibbs Statistical MECHANICS}

On the one hand, Boltzmannian statistical mechanics (BSM) introduces, between the microstatistical and the macrovariable levels, a third level of description ${ }^{6}$ based on the notion of 'macrostate' (Lavis, 2005). On the part that connects it with the thermodynamic description level, a particular macrostate $\Gamma_{\mathrm{m}}$ of the system would be defined from the specific values of a set of macrovariables $f$ that describe the system observationally. On the part that connects it with the level of mechanical description, an individual macrostate $x$ of the system is determined by a continuous set of many microstates, and therefore would be directly linked to a particular region of phase space. In this sense, a set of macrostates $\Gamma_{\mathrm{mi}}$ of a system would constitute in turn a partition of phase space $\Gamma$ into topologically disjointed regions. Therefore, according to BSM the system would be mechanically described by a single macrostate $\Gamma_{m}$ at each time $t$, connected both (a) to the phase region $\Gamma_{m}$ in which the

4 Here lies a conception of statistical mechanical probabilities as 'shortcuts' to effectively compute an overwhelming amount of microscopic data (Shenker, 2020).

5 I am grateful to an anonymous reviewer for mentioning this point.

6 Lavis (2005) established three levels of statistical mechanical description, which I will use in my analysis, namely (1) the microstatistical or dynamic level, (2) the macrostatistical or statistical level, and (3) the thermodynamic level. 
actual microstate $x$ of the system would be found within a continuous set of macroscopically indistinguishable microstates, and (b) with the particular real-value of each of the macrovariables $f i$ that observationally describe the system, thus connecting both levels of description.

It should be noted that the historical introduction of macrostatistical descriptions of physical systems by Boltzmann in his celebrated 1877 combinatorial argument responds precisely to the above problem of the practical impossibility of completing the microstate of the system (Uffink, 2007). Within the Botzmannian literature (Lebowitz, 1993; Albert, 2000; Goldstein et al., 2020), the introduction of macrostates (or also coarse-graining) by means of partitioning phase space $\Gamma$ is usually justified on the basis of the instrumental limits of the observational capacity of scientific agents. In this sense, each of the possible microstates that make up a particular macrostate $\Gamma_{m}$ would be observationally indistinguishable from any other distinct microstate within that same phase region associated with the macrostate. Here, it should be introduced the probabilistic assumption of BSM wherein all the microstates contained in $\Gamma_{\mathrm{m}}$ are equiprobable.

On the other hand, Gibbsian statistical mechanics (GSM) is theoretically based not on the statistical mechanical description of an individual physical system, such as a gas in a vessel, but on a continuous set of virtual copies of the same system subject to constant macroscopic values but with different microscopic values, which is usually called 'ensemble'. Depending on the macrovariables used as observational constrains, the ensemble used to describe the phenomenon will be (mainly) microcanonical, canonical or grand canonical. Those ensembles are represented by a probability distribution function $r$ defined over the phase space $\Gamma$ associated with the system, by which the probability of occurrence of the actual microstate $x$ of the system within the phase region $\mathrm{r}$. In this sense, the values of the macrovariables A characterizing the system at each moment would be obtained by phase averaging $\langle\mathrm{A}\rangle_{\Gamma}$ all macro-values derived from the probability distribution $\mathrm{r}$, thus theoretically connecting the level of observational-thermal dynamic description of the system with its level of microstatistical mechanical description.

\section{Boltzmannian and Gibbsian Accounting for the Approach to Equilibrium}

On the one hand, for BSM the thermal equilibrium state of a system would be properly represented mechanically by a particular macrostate $\Gamma_{m}$ of this system (Lazarovici, 2019). In particular, one of the most extended assumptions within the Boltzmannian approach (defended by Boltzmann [1896] himself) is that this equilibrium macrostate $\Gamma_{\text {meq }}$ is precisely the one with the largest phase volume, where this volume is usually measured by means of Lebesgue's measure, ${ }^{7}$ which is justified by the fact that this measure will be conserved during the dynamic evolution of the system according to Liouville's theorem. ${ }^{8}$ It

\footnotetext{
As Shenker (2020) points out, the constant use of the Lebesgue measure $\mu$ within the literature is usually justified because it is conserved during the evolution of the system according to the Liouville theorem (see note 7).

8 Liouville's theorem states that the $\mu$-measured phase volume of a set of microstates of a system will conserved during its dynamic (and Hamiltonian) evolution.
} 
should be noted that the famous Boltzmann entropy $S_{B}$ of an individual macroscopic system encoded in an individual microstate is defined by the natural logarithm of the measure of the number (or properly 'volume') of microstates contained within the macrostate-region $\Gamma_{\mathrm{M}}$ in which the actual microstate $x$ is located at that time.

$$
\mathrm{S}_{\mathrm{B}}\left(\Gamma_{\mathrm{m}}\right)=k \operatorname{In} \mu\left(\Gamma_{\mathrm{m}}\right)
$$

In the context of BSM, the entropy of a physical system will depend directly on the phase volume of the macro-state in which it is located. Therefore, if the actual microstate $x$ of the system is found within the set of observationally indistinguishable microstates (and with the same probability of occurrence) contained in macrostate $\Gamma_{\mathrm{m}}$, the value of its Boltzmann entropy will increase if that microstate $\mathrm{x}$ dynamically transits towards a macrostate-region $\Gamma_{\mathrm{m} 2}$ whose phase volume is greater than $\Gamma_{\mathrm{m}}$ and will decrease if it transits towards a macrostate-region $\Gamma_{\mathrm{m} 1}$ whose phase volume is lesser than $\Gamma_{\mathrm{m}}$. Thus, an individual system will be BSM-represented as being in its thermal state if its actual microstate $\mathrm{x}$ lies within the macrostate-region $\Gamma_{\text {meq }}$ of largest volume (in fact, $\Gamma_{\text {meq }}$ occupies almost the entire $\Gamma$ ), associated with the macroscopic values of the thermal state.

As is well known, one of the main disadvantages of the Boltzmannian representation of the thermal equilibrium state is that, as derived from the Loschmidt irreversibility paradox and the Poincaré recurrence theorem, there is a theoretical possibility (although ridiculously improbable) that, once the representative microstate is within the region associated with the thermal equilibrium, it may transit towards regions of smaller volume and therefore abandon the equilibrium state. ${ }^{9}$ This, of course, directly contradicts certain empirical principles of thermodynamics, by which (i) every system must progressively approach its state of equilibrium if it is not already in fact in this state (i.e. law of approximation to equilibrium, see Shenker and Hemmo, 2012) and (ii) the entropy of a system can never decrease (i.e. second law of thermodynamics). However, the demonstration at the beginning of the 20th century of the existence of spontaneous thermal fluctuations at the molecular level (where the second law is constantly violated) caused these principles of thermodynamics to be replaced by empirical regularities whose validity was not deterministic but statistical (see Sklar, 1993).

On the other hand, for GSM the thermal equilibrium state of an ensemble is represented statistically by what Gibbs himself called 'statistical equilibrium' and defined as follows: the probability distribution $r$ that represents the ensemble is in statistical equilibrium if and only if it is stationary. Additionally, the phase volume of $\mathrm{r}$ will be conserved during the dynamic evolution of the system according to Liouville's theorem of classical mechanics. Note that through this Gibbsian formulation of the thermal equilibrium state, the average phase values $\langle\mathrm{A}\rangle_{\Gamma}$ of the macrovariables (pressure, temperature, volumen and so on) that observationally describe the ensemble remain invariant once the probability distribution $r$ reaches statistical-ensemble equilibrium. In the same way that Boltzmann (1896) aimed to reformulated Clausius thermodynamic entropy by means of mechanical concepts

9 While the irreversibility paradox states that the reversibility of the system components would allow the systems to have anti-thermal behaviors (e.g., decreasing their entropy), the recurrence theorem states that the actual microstate of the system will return to that same point in phase space in an extremely long period of time (Goldstein, 2020). 
(i.e. molecular position-velocities) and statistical assumptions, the Gibbs fine-grained entropy $S_{\text {Gf }}(2)$ of an ensemble (or just 'ensemble entropy') was straightforwardly defined from the integral of the probability distribution defined over phase space:

$$
\mathrm{S}_{\mathrm{Gf}}[\rho]=-k \int_{\Gamma} \rho \log \rho
$$

However, this implies according to the Liouville theorem of classical mechanics that the Gibbs fine-grained entropy $S_{G f}$ of the $\rho$-encoded ensemble remains constant during the evolution of the system, even when this evolution corresponds to an equilibration process.

Let's suppose that we want to SM-represent a paradigmatic thermodynamic behavior, such as the approximation of certain systems to their thermal equilibrium state or also known as 'equilibrium process'. For example, let us imagine the paradigmatic case of a gas confined in half the volume $\mathrm{V}$ of a vessel, which, after retiring the partition inserted in the middle of the vessel, begins to expand spontaneously until it occupies its entire volume $2 \mathrm{~V}$.

On the one hand, the approximation of a system to its state of thermal equilibrium would be described in BSM, at least in its standard formulation, as follows. Fundamentally, the free expansion of a gas in a vessel would be represented by a phase path goriginated in a macrostate $\Gamma_{\mathrm{m}}$ occupying a small region in $\Gamma$ and directed towards larger phase regions until eventually reaching the phase region associated with the equilibrium macrostate $\Gamma_{\text {meg }}$ at the moment when the gas reaches the entire volume of the vessel in which it expands (Figure 1, left). It is assumed that $\Gamma_{\text {meg }}$ occupies almost the entire phase space $\Gamma$ of the gas-recipient system. Of course, once the phase trajectory ghas reached the region associated with the equilibrium region $\Gamma_{\text {meq }}$ it will be able (with a negligible degree of probability) to transit regions external to that macro-region at later moments of the dynamic evolution of the gas. According to the definition of Boltzmann entropy (1), its value would be modified during the expansion of the gas proportionally to the volume of the macrostate through which the actual microstate $x$ dynamically transits across $\Gamma$, reaching its maximum value once it reaches the thermal equilibrium macrostate $\Gamma_{\text {meq }}$ at $\mathrm{t}$, so that $k \log \mu\left[\Gamma_{\mathrm{m}}\left(t_{0}\right)\right]<k \log \mu\left[\Gamma_{\text {meq }}(t)\right]$, (see Figure 1, left). It is precisely in the system's equilibrium state (i.e. the gas occupies the entire volume of the vessel) wherein the value of Boltzmann entropy will coincide numerically with Clausius thermodynamic entropy (see Callender, 1999).

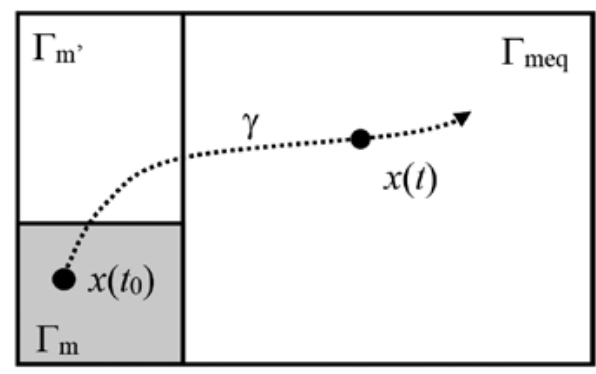

$k \log \mu\left[\Gamma_{\mathrm{m}}\left(t_{0}\right)\right]<k \log \mu\left[\Gamma_{\mathrm{meq}}(t)\right]$

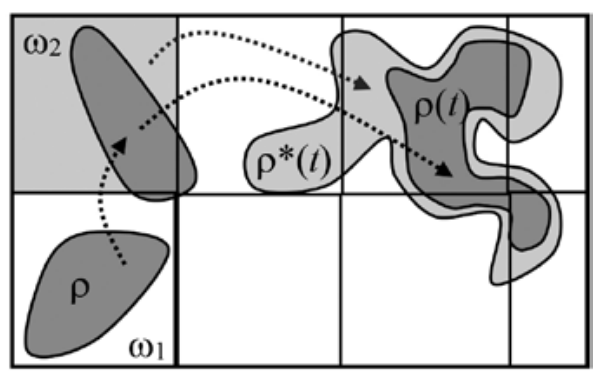

$\mathrm{S}_{\text {Gf }}(\rho) \leq \mathrm{S}_{\mathrm{Gc}}\left(\rho^{*}\right)$

Figure 1

Phase-space portraits of a physical system approaching thermal equilibrium according to BSM (Left) and GSM (Right) 
On the other hand, the fact that the Gibbs fine-grained entropy (2) of the probability distribution $r$ remain constant during the entire expansion of the gas in a closed vessel somehow go against thermodynamic predictions (i.e., the value of the thermodynamic entropy of the gas-recipient must increase during this same process), assuming that the former must somehow be consistent with the empirical results of the former. ${ }^{10}$ To this end, Gibbs (1902) himself introduced an algorithm to allow entropy to 'change' in the dynamic evolution of the system, highly increasing the computational ease with which the values of the macrovariables could be calculated (Uffink, 2007). This algorithm was based on partitioning (also called coarse-graining procedure) the system's phase space $\Gamma$ into cells $\omega \mathrm{w}_{\mathrm{i}}$ having the same phase volume.

Based on this partitioning, at a certain moment of the Liouville-driven dynamic evolution of the distribution $\rho$ over the phase space $\Gamma$ of the gas, a phase average $\langle A\rangle$ of the macroscopic values encoded in the ensemble-represented probability distribution $\rho$ will be performed, thus deriving a probability distribution $\rho^{*}$ whose main distinctive feature is its uniform distribution on $\Gamma$. Once defined this new probability distribution $\rho^{*}$, it can be used to determine the values of a new measure of entropy, usually called Gibbs coarsegrained entropy $S_{G c}\left(\rho^{*}\right)$. In this new coarse-grained context, the theoretical criterion for defining the statistical equilibrium of the ensemble is no longer its stationarity but the degree of uniformity with which its values are distributed. In this way, it is easy to see that $S_{G f}(\rho) \leq S_{G c}\left(\rho^{*}\right)$ (see Figure 1, right), i.e., the value of coarse-grained entropy defined on $\rho^{*}$ will always be greater than or equal to (in the case where both $\rho$ and $\rho^{*}$ are uniform) the fine-grained entropy defined on $\rho$. By means of this precise algorithmic procedure, we can efficiently compute from GSM the approach of the $\rho$-encoded ensemble to the coarsegrained state of statistical-ensemble equilibrium (wherein phase averages quantities $\langle A\rangle$ remain invariant), in a manner consistent with or mimicking the increase in thermodynamic entropy of the gas until it reaches its maximum by occupying the entire volume of the vessel (see Wallace, 2020).

\section{The Foundational Schism between Boltzmann and Gibbs Statistical Mechanics}

As it has been displayed in the previous sections, there is a significative split of the theoretical architecture of SM into BSM and GSM. Although in this section I will go deeper into such splitting, the reader should not forget the common theoretical ground (e.g. use of phase space) (Section 2.1) or the empirical success of both frameworks on the same domain of reality, which makes the splitting of this discipline even more surprising. My main thesis is that this deep theoretical split of SM can be supported, on the one hand, by the fact that (i) neither the central concepts of BSM are interchangeable with those of GSM or vice versa (Section 2.2), (ii) nor the individualist-based descriptions of thermophysical phenomena from BSM can be completely translated into GSM-ensemblist terms (Section 3), independently of whether their numerical values coincide (as usually happens in equilibrium, see Lazarovici, 2019) or not.

10 For assessing the original discussion on the conceptual and quantitative connection between Gibbs entropy and Clausius entropy via 'thermodynamical analogues', see Gibbs (1902). 
Illustratively, Boltzmann concept of thermal equilibrium cannot be interchanged with the Gibbsian concept of equilibrium simply because its way of representing (or its intension') this state is different, since the former defines from macrostate (reportable from GSM) and the latter by properties of a probability distribution $\rho$. Essentially, both concepts are not theoretically equivalent or co-extensional precisely because the state-of-affairs they refer to (or its 'extension') would be radically different in both cases: while Boltzmann equilibrium state refers to a thermal property of an individual physical system, the Gibbsian equilibrium state refers to a statistical property of a fictitious set of virtual copies (namely, the ensemble) of the target system (Goldstein et al., 2020).

On the other hand, the fact that the statistical mechanical concepts of BSM and GSM are not interchangeable consequently means that the descriptions of thermal phenomena which can be derived from both theoretical devices, as in the case of a gas expanding spontaneously through the volume of a vessel (see Section 3.2), cannot become interchangeable. One of the most illustrative cases of this theoretical untranslatability between BSM and GSM is what is known in the literature as 'Sklar's dilemma' (Sklar, 1993; McCoy, 2020), which can be described for the case of the equilibrium approach of the systems as follows. While for the Gibbsian approximation, once the ensemble $r$ reaches the statistical-ensemble equilibrium state (by means of the coarse-graining procedure, as detailed above) it will remain there if there is no external intervention, according to thermodynamic predictions. For the Boltzmannian approximation, once the individual system gradually reaches the macrostate-region $\Gamma_{\text {meq }}$ of thermal equilibrium, it may eventually fluctuate outside that equilibrium macrostate, against the empirical regularities of thermodynamics.

However, the fact that the two main approaches to one of the central disciplines of modern physics are theoretically disconnected poses a huge problem for the foundational task of understanding the mutual co-existence of two SM-theories (not even two SM-formalisms or SM-interpretation) about the very same physical domain. This fundamental theoretical split of SM has recently been treated interpretively by Frigg and Werndl (2019) in such a way that BSM and GSM would constitute different types of statistical mechanical theories. Namely, the first as a fundamental theory and the second as an effective theory. This means that "BSM provides a true description of the system within the scope of SM; GSM offers an algorithm to calculate values defined by the fundamental theory" (Frigg and Werndl, 2019, p. 425). However, that the Boltzmannian approach can be conceived as a 'fundamental' theory does not imply that the GSM theoretical apparatus can be derived entirely from BSM, nor that the epistemic virtues of the latter can be inherited by the former. Claiming that BSM is a 'fundamental' theory just means that the macroscopic properties of the systems are assumed within this framework to 'supervene' (or 'depend directly') on the basic mechanical properties of the molecular system (e.g. Shenker, 2020).

On the other hand, the ensemblist and pro-GSM philosopher David Wallace (2020) proposes to interpret $\mathrm{BSM}$ not as a different domain à la Frigg-Werndl, but as a special case of GSM. However, as the author himself points out (Wallace, 2020, pp. 584-585), this does not imply that both should be characterized from the same theoretical resources, since it is evident that this is not the case, i.e. BSM: macrostates, individual systems, Boltzmann entropy (1), Boltzmann equilibrium, and GSM: phase averages, ensembles, Gibbs fine-grained (2) and coarse-grained entropy, and equilibrium ensembles. Although it is true that Gibbs entropy mathematically generalizes Boltzmann entropy for non-uniform probability distributions over microstates, the latter incorporates in its definition the notion of 
system's macrostate (set of observationally indistinguishable micro-states), which is meaningless within standard GSM's ensemble-based theoretical apparatus. Therefore, that according to Wallace (2020) BSM should be regarded a special case of GSM regarding the application domain can be fully compatible with both empirically successful SM-approaches using different concepts and generating different theoretical descriptions of the same phenomenon (Figure 1). This theoretical-conceptual separation is well illustrated in this quotation of Goldstein:

The Gibbs entropy [2] is an efficient tool for computing entropy values in thermal equilibrium when applied to the Gibbsian equilibrium ensembles $\rho$, but the fundamental definition of entropy is the Boltzmann entropy [1]. We have discussed the status of the two notions of entropy and of the corresponding two notions of thermal equilibrium, the "ensemblist" and the "individualist" view. Gibbs's ensembles are very useful, in particular, as they allow the efficient computation of thermodynamic functions (...), but their role can only be understood in Boltzmann's individualist framework. (Goldstein et al., 2020, p. 576)

Following Goldstein here, I defend now that the statistical mechanical schism existing between BSM and GSM (as well as between 'individualists' and 'ensemblists', see Goldstein, 2019) must be fundamentally understood as a split of the main epistemic functions (e.g. explanatory, predictive, etc.) of SM globally considered. In particular, I argue that BSM is a statistical mechanical theory capable not only of providing true descriptions but also of generating powerful explanations of certain phenomena; while GSM is a statistical mechanical theory capable of generating effective predictions about the evolution of the macrovariables of the observed system. In the following, I will focus, on the one hand, on defining how we should conceive this 'explanatory power' of BSM and 'predictive effectiveness' of GSM; and, on the other hand, on explaining the main arguments why these two statistical mechanical devices should be theoretically qualified according to these epistemic capabilities.

\section{The Explanatory Power of Boltzmann Statistical Mechanics}

It is openly assumed within the scientific community that one of the main tasks of SM is to explain the macroscopic behavior of certain systems from the inaccessible dynamics of their components. In particular, this discipline must explain what phase changes are experienced by certain materials (i.e. why an ice melts into a hot cup), why certain properties of a fluid remain stable under certain conditions or why a gas tends to expand spontaneously throughout the volume of the container that contains it. In short, SM could be assumed as a fundamentally explanatory physical theory.

Although this explanatory character of SM is widely recognized, we do not find in the literature any systematic and explicit discussion on how we should philosophically understand such kinds of explanations, at least until the recent publication of 'Statistical Mechanics and Scientific Explanation' (Allori, 2020). It should be noted here that virtually all previous philosophical analyses of statistical mechanical explanations employed an everyday notion of 'explanation', with exceptions like Myrvold (2016) or Wilhelm (2019), who seem to explicitly advocate a statistical and a deductive-nomological model of explanations, 
respectively. For reasons of extension, in this paper I will follow this strategy ${ }^{11}$ Specifically, to define the concept of 'explanatory power' I will not presuppose any of the main models of explanation (i.e., causal-mechanistic, unificationist, pluralist, etc.), although it may arguably be more compatible with one than with the other. Illustratively, Lazarovici and Reichert defended that the application of deductive-nomological model in this scenario (consisting of the logical-deductive derivation of macroscopic laws from microscopic laws and auxiliary assumptions) would be especially problematic precisely because this "misses the crucial role that initial conditions play in the explanation of a macroscopic phenomenon" (Lazarovici and Reichert, 2015, p.704). In the vein of Lazarovici and Reichert (2015), the only minimalist requirement would only be that any statistical mechanical explanation must at least appeal both to (i) the system's initial conditions at $\mathrm{t}_{0}$ and (ii) a property $\mathrm{P}$ about the dynamical behavior of the system's molecular constituents, since this is precisely the characteristic feature of such a physical discipline (see Section 2.1). Note that these two minimum requirements are prima facie compatible with both BSM and GSM, since it is only required that the explanation of the thermal-macroscopic behavior of a substance descriptively depends on the dynamics of its molecular components (Section 2).

Interestingly, Myrvold (2016) recently developed an account on SM-explanations of the approach to equilibrium based in these two minimal requirements (i-ii): "A system behaves as it does because of its dynamics, together with initial conditions. Explanations of relaxation to equilibrium will have to involve an argument that the dynamics, together with initial conditions of the right type, yields that behavior." (Myrvold, 2016, p. 33). In this quotation, Myrvold points out that it is precisely (i) a microstatistical-dynamic property and (ii) certain particular initial conditions ${ }^{12}$ that would make up the explananda of the equilibrium approach of a gas as an explanandum. Thus, this plausible statistical mechanical explanation would also require explaining (or at least justifying consistently) why this particular dynamic is somehow theoretically and experimentally compatible with the initial conditions proposed to describe the values of the system at the beginning of this evolution. Up to this point, a minimal explanatory power criterion can be defined as follows

\section{Explanatory Power Criterion:}

A statistical mechanical description $\mathrm{D}$ of the thermal behavior $\theta$ of a molecular system constitutes a powerful explanation of $\theta$ if this description is abducted from (i) the system's (either ensemble $\rho$ or macrostate-encoded $\mathrm{M}_{0}$ ) initial conditions and (ii) the microstatistical properties $\mathrm{P}$ of the system that generates during its evolution such thermal behavior $\theta$.

As it can be noticed, the main virtue of this criterion is that it offers us a guarantee that the content of the explananda (i.e., a thermal behavior as the approximation to equilibrium) would be abductively derived according to the procedures of the respective statistical mechanical formalism of the content of the explanadum, being this (i) the initial condition and (ii) a microstatistical property of the system that gives rise to that macroscopic

${ }^{11}$ I will leave for a future paper the task of analyzing the compatibility of statistical mechanical explanations with the models of scientific explanation that we can find in the philosophical literature.

${ }_{12}$ For instance, by assuming that the system would initially be properly represented by a uniform probability distribution à la Albert (2000). 
behavior. I agree with Lazarovici and Reichert (2015, p. 704) in defending that a mere formal-deductive derivation of the equilibrium from (ii) a particular dynamic property of the system would not constitute properly an 'explanation' of such a thermal phenomenon, since this would trivialize the importance of the initial/boundary conditions of the system. That is why this criterion is based on abductive reasoning, where the approach to equilibrium could be inferred (in an approximate, estimated and fallible way) from the behavior of the system's components and always according to the particular circumstances. Another advantage of this explanatory power criterion in SM that I defend is directly part of the pivotal consideration of statistical mechanics, wherein the thermal behavior of the systems to be explained depends intrinsically on the deterministic and reversible evolution of the system components (common assumption from both BSM and GSM, see Section 2). Finally, in this criterion I postulate at least a minimal necessary condition for a description to constitute a powerful explanation; this would allow to be philosophically complemented by additional necessary conditions, although it is highly debatable what these should be.

On the one hand, one can show how any standard description of the paradigmatic thermal behavior of the GSM-derived equilibrium approach (Section 3.2) would not meet the explanatory power criterion: i.e., "the ensemble interpretation of statistical mechanics makes it unclear how statistical mechanics can explain the behavior of individual systems" (McCoy, 2020). The main reason is twofold. Firstly, the Gibbsian descriptions of thermal-macroscopic behavior (see Section 3) do not specify any microstatistical properties from which can be abductively derived the values of the macrovariables $f_{\mathrm{i}}$ encoded in the coarse-grained probabilities ${ }^{13} \rho^{*}$, they simply specify the theoretical procedures required to adjust (via phase-averaging and coarse-graining) these values according to phenomenological thermodynamics. Thus, standard GSM-descriptions fail to satisfy condition (ii) of the above minimum criteria. Second, these same Gibbsian descriptions depend constitutively on eliminating the microstatistical details pertaining to the initial system condition (encoded in $\rho$ ) by introducing a new probability distribution $\rho^{*}$. Therefore, they would also fail to satisfy condition (i). Therefore, this criterion would shed light on why GSM descriptions not only do not constitute powerful explanations, but have no explanatory capacity regarding equilibration, a fact agreed in the SM community as noted above. In Wallace's words: "As such, despite the acknowledged technical advantages of the Gibbsian approach, the Boltzmannian offers the true explanation of the success of statistical mechanics" (Wallace, 2020, p. 584).

In fact, Wallace $(2011,2020)$ sought to exploit the explanatory potential of GSM to the fullest. On the one hand, his proposal satisfies condition (ii) by specifying the micro-dynamical properties of the system through what he calls 'Simple Dynamical Conjecture $^{14}$ ' (Wallace, 2011). On the other hand, Wallace's proposal also initially satisfies (i) by specifying in density $r$ the initial condition of the system at $t_{0}$. However, his 'Simple Dy-

${ }^{13}$ Outside the Gibbsian context, some pro-BSM authors defend (even in an explanatory way) that the dynamics of the system must have some fancy mathematical properties such as 'ergodicity' or 'mixing', by which the microstates of a small phase region would eventually cover all the space of a larger phase region (see Sklar, 1999).

14 "Simple Dynamical Conjecture (for a given system with coarse-graining C): Any distribution whose structure is at all simple is forward predictable by C; any distribution not so predictable is highly complicated and as such is not specifiable in any simple way except by stipulating that it is generated via evolving some other distribution in time" (Wallace, 2011). 
namical Conjecture' necessarily requires introducing at $t_{1}$ a coarse-graining $C: \rho \rightarrow \rho^{*}$ of the initial density $r$ to a new density $\rho^{*}$ with certain properties (like forward-compatibility, see Robertson, 2020). Thus, Wallace's $(2011,2020)$ Gibbsian explanation of the approach to equilibrium ceases to satisfy (i) when coarse-graining at is introduced at $t_{1}$, due to the fact that the required initial conditions (i) are no longer specified in $\rho^{*}$. Therefore, it does not satisfy the explanatory power criterion.

On the other hand, one can argue that certain BSM descriptions of thermal behavior can meet the above explanatory power criterion. In fact, Boltzmann himself (1896) was one of the pioneers in providing a statistical mechanical explanation of this thermal phenomenon. According to his canonical proposal (Callender 1993), the approach to equilibrium could be powerfully explained from (i) the fact that every possible microstate $x$ contained in low-entropy macrostate $\Gamma_{\mathrm{m}}$ transits 'typically' (i.e., except in an insignificant number of cases, see, Lebowitz, 1993) towards the equilibrium macrostate $\Gamma_{\text {meq }}$, and from (ii) the fact that the system is actually within a low-entropy macrostate $\Gamma_{m}$ (Figure 1, left). In terms of Goldstein et al. (2020) "a feature or behavior is said to be typical in a set $S$ if it occurs for most (i.e., for the overwhelming majority of) elements of S" (Goldstein et al., 2020, p. 559). This would constitute a powerful explanation according to the above criterion. Boltzmann's original explanatory strategy will be recovered from the 1990s onwards by Neo-Boltzmannian authors such as Lebowitz (1993) or Goldstein (et al., 2020) as the 'typicalist explanation' of the approach to equilibrium based on the abductive exploitation of the vast majority of the phase volume from the initial macrostate ${ }^{15} \mu\left(\Gamma_{m}\right)$ it would be within the equilibrium macrostate-region $\Gamma_{\text {meq }}$ after a certain time interval, except for an extremely tiny subvolume that would account for thermal fluctuations outside equilibrium.

The key here is that this distinctive type of typicalist reasoning (Wilhelm, 2019) allows us to delimit with conceptual precision what is the plausible microstatistical or molecular-dynamic reason (or 'hypothesis', in abductive terms) that underlies macroscopic behaviors such as gas equilibration within a vessel. Recently, Wilhem (2019) argued from a Hempelian framework of explanations that typicalist reasoning is not necessarily probabilistic (or 'inductive'). In this sense, I defend that the inferential behavior underlying typicality-based explanatory reasoning would be nicely characterized as being an 'abductive' (not deductive nor inductive) process:

Initial Condition (i): $\quad$ The actual microstate $x$ is in $\Gamma_{\mathrm{m}}$ at $\mathrm{t}_{0}$ Typicality Hypothesis (ii): Most microstates in $\Gamma_{\mathrm{m}}$ at $\mathrm{t}_{0}$ will be in $\Gamma_{\mathrm{meq}}$ at $\mathrm{t}_{1}$

Abducted Explananda: $\quad$ It is plausible that the actual microstate $x$ is in $\Gamma_{\text {meq }}$ at $\mathrm{t}_{1}$

Therefore, (ii) 'typicality' as a microstatistical property together with (i) certain ideal initial conditions (like an initial low-entropy macrostate $\Gamma_{m}$ or 'Past Hypothesis', Albert, 2000) would allow us to powerfully explain within BSM the approach to the equilibrium of macroscopic systems from the behavior of their microscopic components (Lazarovici and Reichert, 2015, p. 704). Other pro-BSM authors postulate that statistical mechani-

15 The phase volume of each macrostate would be determined by means of the Lebesgue measure, which is conserved during the evolution of the system according to the Liouville principle. However, this is not the only measure of phase volume we could consider (see Shenker, 2020). 
cal explanations of equilibrium should not be based on typicality as a physically significant microstatistical property but on certain mathematical properties of dynamical systems, such as the explanation by Frigg and Werndl (2011) based on what is known as epsilon-ergodicity. However, the physically significance of such formal-dynamic ergodic-like properties (namely, whether these properties can be applied to real molecular systems) is still contentious within the philosophical literature.

In any case, the explanatory potential of the Boltzmannian theoretical apparatus rests directly on the descriptive exploitation of the microstatistical dynamics of 'individual' physical systems allows it under certain conditions to generate theoretically powerful explanations of thermal behaviors such as the expansion of a gas in a vessel, making abductively explicit the microstatistical properties (i.e. typicality) that produce such behaviors. Then, BSM stands as a physical theory well-capable of generating powerful statistical mechanical explanations of certain thermal behaviors.

\section{The Predictive Effectiveness of Gibbsian Statistical Mechanics}

In contrast to the descriptive-explanatory virtues that I have just pointed out in detail in the previous section, Boltzmannian formalism has been enormously disregarded throughout the history of statistical mechanical practices due to its expensive calculus-computational costs when using it in real scientific contexts. Among these practices, the prediction of observational values during the dynamic evolution of certain systems would be undoubtedly one of the central epistemic functions of SM. However, for BSM (although its many real applications) it would be difficult to efficiently compute such extremely complex $\left(\mathrm{n} \approx 10^{23}\right)$ predictions. This would somehow explain the hegemony of GSM during the 20th century: "Surely, the calculational ease of the Gibbs approach is the primary reason why it dominates other approaches" (Callender, 1999, pp. 349, 354-355), at least until the explanatory-conceptual value of BSM started to be vindicated in the 90s.

As far as predictions are concerned, there is a consensus within the philosophical literature about SM (Wallace, 2020) about the enormous effectiveness of Gibbsian formalism in calculating future values of macrovariables of systems with thermal behavior. In this sense, I must now point out that another of the fundamental epistemic virtues of statistical mechanics is not the possibility of developing powerful predictions but its capacity to generate properly effective pragmatic predictions. Namely, that those predictions can be carried out by means of techniques and resources realistically accessible to the majority of competent physicists. Note that this is not merely a pragmatic virtue, but also an epistemic one, since the possibility of carrying out statistical-mechanical predictions with technical ease determines the knowledge about the phenomena terms that we can acquire through SM. From this, a second criterion can be defined, stablishing the minimum necessary conditions for a prediction to be an effective one:

\section{Predictive Effectiveness Criterion:}

A description of the future macrovariables $f_{\mathrm{i}}$ of a system with thermal behavior $\theta$ constitutes an effective prediction if (i) the computation of such values can be computationally treatable, and in that case (ii) if such description specifies a well-defined algorithmic procedure that allows calculating such values by means of realistic computational resources with respect to actual practices. 
Again, this criterion proposes a necessary-minimalist condition (again, it could be complemented by further conditions) for a statistical mechanical prediction to be effective: simply, that it can be carried out by means of realistic computational resources-procedures, and if this is the case, that such computational procedures are specified through a well-defined algorithm. I must make it clear that the lack of predictive effectiveness of a theoretical device does not mean that it does not have multiple (and even solid) practical applications in various fields. For example, the fact that BSM can develop predictions about how a liquid crystal will evolve in contact with a heat bath does not mean that this prediction is 'effective' in the sense specified above.

For the Boltzmannian program, to be able to predict the macroscopic values that the system will take in future moments of the dynamic evolution it will be prima facie necessary, in the first place, to determine the number of microstates contained within the macrostate $\Gamma_{\mathrm{m}}$ wherein the actual microstate $x$ of the system would be located at the beginning of its dynamic evolution. As I claimed in Section 2, this is not only a computationally exhaustive task (approximately $10^{23}$ position-velocity values per individual microstate), but also computationally intractable since the number of microstates $\mu\left(\Gamma_{\mathrm{m}}\right)$ to be determined is continuously infinite, see Figure 1. Even if we ideally can solve this problem (for example, by means of discretization techniques or an ad hoc coarse-graining), we must also solve the mechanical analytical equations of motion associated with each microstate represented by phase trajectories. However, as Shenker points out: "This is an idealization, and such calculation is doubly impossible: the system is too complex, and the number of microstates, and hence of trajectory segments, is a continuous infinity" (Shenker 2020, p. 11). Note that even the definition of the probability that a microstate will be in a particular macrostate at a particular time ${ }^{16}$ depends also on carrying out these computationally impossible calculations.

Although explanatorily powerful and conceptual consistent, most of Boltzmannian predictions of physically significant systems (a number of components of $\mathrm{n} \approx 10^{23}$ ) do not meet the above effectiveness criterion based on mathematical tractability and pragmatic feasibility. Additionally, BSM does not specify any 'canonical' or 'standard' algorithmic procedure to perform predictive calculations in a pragmatically realistic manner with respect to those available to scientific agents in actual statistical mechanical contexts. I could argue at this point that the main reason underlying BSM's inability to predict is precisely its epistemic dependence (descriptive, explanatory and predictive) on individual microstates as fundamental representational structures; however, as McCoy points out: "Individual microstates, in contrast to macrostates, are essentially irrelevant to making predictions in the theory" (McCoy, 2020). In order not to convey the wrong impression to the reader, I will stress once again that, despite not being a predictively effective theory according to this criterion, BSM has an extremely extensive domain of predictive applicability ranging from ideal gases to Kerr black holes. But the central issue here is that those predictions are both calculationally difficult to perform and extremely computationally expensive.

On the other hand, it could be argued that in the context of GSM, descriptions of future macrovariables of the system would generally be assumed as effective SM-predictions

${ }^{16}$ This probability value is defined in terms of the proportion of microstate from the initial macrostate that are in the region associated with the latter macrostate. 
because of the role of statistical techniques like coarse-graining and phase averaging. In this sense, Malament and Zabell claimed that "As a black box technique, Gibbs phase averaging works just fine. The question is why it works" (Malament and Zabell, 1980, p. 340). First, many Gibbsian-ensemblist authors like Wallace (2020) justify the introduction of probabilistic techniques like phase averaging and coarse graining based on the avoidance of computationally expensive microstatistical calculations typical of Boltzmannian predictions. This grounds precisely what Wallace (2020) calls the (we would add 'pragmatic') necessity of GSM. Other authors such as Malament and Zabell (1980) justify these Gibbsian procedures on the assumption that the target system is ergodic. Disregarding how these techniques can be justified, they play a pragmatically indispensable role in SM-practices: "[probabilistic techniques] are shortcuts that enable practical predictions, which are actually carried out in statistical mechanics" (Shenker, 2020, p. 11).

Secondly, such Gibbsian predictions are not only realistically treatable, but also specify an algorithmic procedure to practically predict, for example, what certain observable values of a gas will be when it has already expanded throughout the volume of the vessel from the values measured at the beginning of the evolution: e.g. (1) represents the initial observable values of the gas by means of a (usually canonical) $\rho$-encoded ensemble, (2) allows the probability distribution $\rho$ to transit phase space $\Gamma$, (3) performs a phase average over $\rho$ with respect to the cells of the coarse-grained phase space, deriving a new probability distribution $\rho^{*}$, (4) uses this new distribution $\rho^{*}$ to calculate the future values of the observables of the gas. Therefore, the inability of Gibbsian formalism to specify the microstatistical dynamic properties (recall Section 5) is compensated by its ability to generate practically advantageous computational procedures.

Therefore, the predictive potential of the Gibbsian apparatus is based on the exploitation of practical statistical techniques, mainly in calculating observable values of physical systems. It allows GSM to generate significatively effective predictions of thermal behavior such as the expansion of a gas in a vessel, specifying by means of practical algorithms how this should be realistically carried out. Thus, GSM stands as a physical theory capable of generating effective statistical mechanical predictions of certain thermal behaviors.

\section{Epistemic Schism and Hempel's Symmetry Thesis}

To sum up, I have just evaluated in the last two sections in what sense the distinction between BSM and GSM as two fundamentally different statistical mechanical formalisms should be understood not only as a fundamental theory and an effective theory, respectively (Lazarovici, 2019; Frigg \& Werndl, 2019), but properly as an epistemic schism within SM. Thus, each of these theoretical proposals would fulfill only one of the two main key epistemic functions in the statistical mechanical context, either to powerfully explain (BSM) or to effectively predict (GSM) thermal behavior, but not the other function.

\subsection{Statistical Mechanics against Hempel's Symmetry Thesis}

Interestingly, this epistemic schism between the main approaches to statistical mechanics that I have defended throughout this paper constitutes a clear counterexample to what 
is known in the literature as the 'symmetry thesis' (Douglas, 2009; Fetzer, 2017), which would affirm in broad terms (i) that every satisfactory explanation also has predictive potential regarding the phenomenon explained and (ii) that every satisfactory prediction will have explanatory potential with respect to the predicted values. This was originally defended by Carl Hempel (1958) in his famous text "The theoretician's dilemma", wherein "Hempel was not only defending the position that every adequate scientific explanation is potentially predictive but also the position that every adequate scientific prediction is potentially explanatory" (Fetzer, 2017, Section 5.1). However, such symmetry between explanations and predictions (and even retrodictions, namely, inferring past values) is based, in Hempelian terms, on the fact that both share the same logical form. According to this author, the proper difference between the two lies in the fact that "[an explanation] will qualify as a prediction only if [its explanandum] refers to an occurrence at a time later than that at which the argument is offered" (Hempel 1958, p. 38).

However, as I have shown in detail in the previous sections, a description $\mathrm{D}_{1}$ underlying a powerful statistically mechanical explanations ${ }^{17}$ such as Boltzmann's (1896) or certain typicality-based Neo-Boltzmannian's (see Lazarovici and Reichert, 2015) of the thermal behavior of a gas expanding freely in a vessel won't constitute an effective prediction of the future observable thermodynamic values $f i$ that the gas will take, precisely because the extreme difficulty (if not pragmatic impossibility) of calculating such values from the intractable microstatistical evolution of the system. In the same vein, another description $\mathrm{D}_{1}$ underlying an effective SM prediction of these same values that the gas at its thermal equilibrium must necessarily obviate, by means of pragmatic statistical calculation procedures, the enormous amount of microdynamic data required to explain the process of balancing from the molecular components of the gas. It should be noticed that when Hempel (1956) speaks of 'prediction', he does not explicitly consider the relevance of the pragmatic factors underlying the possible predictions. However, being charitable with the applicability of this Hempelian logical-empiricist position, I should assume that it somehow intended to clarify real scientific practices. In this case, the contingent calculative limitations underlying BSM's predictive ability should also be included in any philosophical assessment (and that includes Hempelian evaluations) of the epistemic dimension of these theories.

In conclusion, one face a fundamental dilemma regarding the epistemic functions of SM: either the statistical mechanical apparatus is conceptually consistent enough to generate descriptions that powerfully explain thermal phenomena from the microstatistical dynamics of their constituents (BSM-horn), or the statistical mechanical apparatus is pragmatic enough to generate descriptions that efficiently predict thermodynamic values (GSM-horn). When a SM-description like $\mathrm{D}_{1}$ satisfactorily meets the necessary condition of the minimalist criterion to constitute a powerful explanation (i.e. specify both the initial macro-condition and the microstatistical dynamic property from which the thermal behavior is derived), then this $\mathrm{SM}$-description $\mathrm{D}_{1}$ won't be able to satisfactorily meet the neces-

${ }^{17}$ Of course, when Hempel (1958) originally developed the symmetry thesis, the type of explanation he used to define that thesis was those based on the famous deductive-nomological model. In any case, I will assume here that the content of such a thesis is generalizable to any model of statistical mechanical explanation. 
sary condition of the minimalist criterion to constitute an effective prediction (i.e. computational tractability and algorithmically specify practical procedures to calculate observable values), and the other way around. That is, according to my analysis developed so far, any SM-description can satisfy either the 'explanatory powerfulness criterion' or the 'predictive effectiveness criterion', but in no case both simultaneously. Therefore, at least in the case of statistical mechanics, the explanatory potential of a theory or a description of that theory does not correlate directly with the predictive potential (in terms of practical effectiveness) of that same theory, against Hempel's (1958) symmetry thesis.

\subsection{Conceptual Architecture and the Foundations of Statistical MECHANICS}

Although I argued that the epistemic schism of SM would be a straightforward contradiction of Hempel's symmetry thesis, I also claim the Hempelian idea by which explanations and predictions of a theory share the form of their logical structure. An assessment of the common structure between the explicability and predictability of theories would allow us, as pointed out by authors such as Douglas (2009), to understand satisfactorily the explanatory value that resides in the predictions, as well as the predictive value that we can find in the explanations. Concerning our aim here, is precisely this that would explain the fundamental (although historically contingent) epistemic split of this very physical discipline. I suggest that what Hempel called 'logical structure' in his 1958 paper could be properly understood in more philosophically fruitful terms (and detached from the assumptions of this author's received view) as the 'conceptual architecture' underlying the explanatory potential or the predictive capacity of BSM and GSM, respectively.

Regarding the conceptual architecture of the theoretical Boltzmann apparatus, its explanatory power and predictive inefficiency would be satisfactorily explained by two elements: (a1) its capacity to descriptively exploit individual microstates $x_{i}$ of the system (see Figure 1, left), which directly appeals to the behavior of the system's components, but does not contribute to the prediction of macroscopic values fi (see McCoy, 2020); and (b1) the coarse-grained partitioning of $\Gamma$ into macrostates $\Gamma_{\mathrm{m}}$ whose direct function is not to easily compute but to microstatistically represent thermodynamic values (Section 2.2). On the other hand, the explanatory deficiency and predictive efficiency of the Gibbsian apparatus would depend intrinsically on certain elements underlying its theoretical architecture: (a2) its capacity to exploit the observable macrovariables ensemble-encoded, predictively efficient but useless to microscopically explain thermal phenomena; and (b1) the coarsegrained of $\Gamma$ in equal cells $\omega_{i}$ (see Figure 2, right) that allow not to represent faithfully but to compute enormous amounts of microstatistical information by means of phase averaging (Section 2.2.). I defend that it is precisely these elements (a1-b2) of the SM conceptual architecture on which the explanatory power (a1 and b1) and the predictive efficiency (a2 and b2) of this discipline are based (see Table 1 below). Consequently, it is precisely the fact that (a1) is theoretically incompatible with (a2) and that (b1) is theoretically incompatible with (b2) that would ultimately explain the epistemic split between BSM and GSM. 
Table 1. Conceptual Architecture of BSM and GSM underlying the Epistemic Schism of SM

\begin{tabular}{ccc}
\hline & BSM & GSM \\
\hline Descriptive element & Individual microstates $x$ & Probability density $\rho$ \\
\hline Partition of $\Gamma$ & Macrostates $\Gamma_{\mathrm{m}}$ & Cells $\omega_{\mathrm{i}}$ \\
\hline Partition Function & Represent macrovariables $f_{\mathrm{i}}$ & Compute phase averages $\langle\mathrm{A}\rangle$ \\
\hline Epistemic & Microstatistical explanatory & $\begin{array}{c}\text { Predictive computational } \\
\text { efficiency }\end{array}$ \\
\hline Virtue & power &
\end{tabular}

However, my defense of the epistemic schism of SM should not be taken as a negative thesis about the foundations of this discipline, but as a recognition of the development during more than one hundred and twenty years of two SM-theories centered on two clearly differentiated epistemic functions: that of powerfully explaining thermal phenomena from their micro-components and that of effectively predicting from a huge amount of microstatistical values. For instance, Boltzmann entropy should not be foundationally conceived as being merely theoretically (or 'numerically') different or equivalent to Gibbs entropy, but properly from the capacity of the former to explain microstatistically the notion of thermodynamic entropy (Callender, 1999) against the practical effectiveness of the latter to calculate future values: "Physicists tend to be busy people; they want and need to obtain practical results about experiments and observable phenomena, and in order to do so they use the best tools at their disposal. For statistical mechanics, the tools are ensemblist tools, pioneered by Gibbs" (Goldstein, 2019, p. 456).

Although both theories have historically evolved along parallel paths, they are not at odds with each other, as Wallace (2020) argues. The thesis that I have defended in this paper can contribute to understanding how the explanatory powerful conceptual architecture (i.e. Hempel's logical structure) of BSM can be fundamental in accounting for the lack of explanatory power of GSM, just as the predictively effective technical apparatus of GSM can illuminate the practical difficulties that BSM entails. Therefore, if we consider statistical mechanics itself as the rich theoretical-conceptual space based on the Boltzmannian and Gibbsian proposals, then this discipline will be globally as explanatory powerful as the first one and as predictively effective as the second one: "the point of $[\mathrm{SM}]$ is (...) an effective framework for the description of complex systems that requires some degree of pragmatism and good physical sense" (Lazarovici, 2019, p. 793). This leads us to think that only by delving into the space opened by the schism between these two great statistical mechanical programs can we continue to progress (as we have been doing during the last decades) in the rich foundations that underlie this fruitful physical theory.

\section{Conclusion}

In this paper I have argued that statistical mechanics cannot be understood univocally as a single theory, but as two fundamentally different theoretical devices, BSM and GSM, based on different conceptual resources and technical-formal procedures. Particularly, my 
main objective has been to argue that the fundamental difference between these two statistical mechanical formalisms must be properly expressed in terms of the epistemic capabilities of both. While Boltzmann's proposal has the ability to generate powerful explanations of thermal phenomena from the dynamics of their components, Gibbs' proposal has the ability to predict in a highly effective way the values of the observables of thermal systems. That is, either one can powerfully explain from BSM why a gas approaches equilibrium when it expands freely in a vessel by appealing to the dynamics of its microscopic components, or one can computationally-efficiently predict from GSM the macroscopic properties that the gas will adopt as soon as it occupies the entire volume of the vessel. Therefore, contrary to Hempel's symmetry thesis, the epistemic ability of a theoretical device such as BSM or GSM to powerfully explain does not correlate (at least directly) with the epistemic ability of this same theoretical device to effectively predict.

By assuming such a split of the main epistemic functions (mainly, explaining and predicting) of the statistical mechanics between BSM and GSM as a constitutive part of this physical theory, we can come to understand in depth why the latter formalism is hegemonic within real predictive practices (Wallace, 2020) and the former is constantly claimed in philosophical domains (e.g. Callender, 1999; Albert, 2000) for its conceptual coherence in providing explanations of thermal phenomena, shedding light on such central foundational questions as "how can one use one formalism to explain the nonequilibrium behaviour of physical systems and to give a foundational account of SM, while continuing to use the other formalism for everyday equilibrium calculation?" (Frigg and Werndl, 2019, p. 425). In conclusion, my proposal to deepen the explanatory-predictive split of statistical mechanics is properly an opportunity to understand that the deep epistemic richness of this physical discipline lies precisely in a desirable close collaboration between the Boltzmannian and the Gibbsian program.

\section{Acknowledgements}

I am very grateful to two anonymous reviewers for helpful comments on earlier drafts. This work has been funded by the Spanish Ministry of Education (FPU16/0774), and it was developed as part of the Research Group in Analytic Philosophy, LOGOS, the Barcelona Institute of Analytic Philosophy (BIAP) and the research project 'Laws, explanation and realism in physical and biomedical sciences' (FFI2016-76799-P).

\section{REFERENCES}

Albert, D. Z. (2000). Time and Chance. Cambridge: Harvard University Press.

Allori, V. (ed.) (2020). Statistical Mechanics and Scientific Explanation: Determinism, Indeterminism and Laws of Nature. London, World Scientific.

Boltzmann, L. (1896). Lectures on gas theory. Berkeley: University of California Press.

Callender, C. (1999). Reducing thermodynamics to statistical mechanics: The case of entropy. Journal of Philosophy 96 (7), 348-373.

Douglas, H. (2009). Reintroducing prediction to explanation. Philosophy of Science 76 (4), 444-463.

Fetzer, J. (2017). Carl Hempel, The Stanford Encyclopedia of Philosophy, Edward N. Zalta (ed.), URL = $<$ https://plato.stanford.edu/archives/fall2017/entries/hempel/>. 
Frigg, R. \& Werndl, C. (2011). Explaining Thermodynamic-Like Behavior in Terms of Epsilon-Ergodicity. Philosophy of Science 78 (4), 628-652.

Frigg, R. \& Werndl, C. (2019). Statistical Mechanics: A Tale of Two Theories. The Monist 102 (4), 424-438.

Goldstein, S. (2019). Individualist and Ensemblist Approaches to the Foundations of Statistical Mechanics. The Monist, 102, 439-457.

Goldstein, S. Lebowitz, J, Tumulka, R. \& Zanghi, N. (2020). Gibbs and Boltzmann Entropy in Classical and Quantum Mechanics. In Allori, V., (Eds.), Statistical Mechanics and Scientific Explanation Determinism, Indeterminism and Laws of Nature-World Scientific. (pp.519-581) London, World Scientific.

Hempel, C. (1958). The theoretician's dilemma: A study in the logic of theory construction. Minnesota Studies in the Philosophy of Science 2, 173-226.

Lavis, D. A. (2005). Boltzmann and Gibbs: An attempted reconciliation. Studies in History and Philosophy of Science Part B: Studies in History and Philosophy of Modern Physics 36 (2):245-273.

Lazarovici, D. (2019). On Boltzmann versus Gibbs and the Equilibrium in Statistical Mechanics. Philosophy of Science 86 (4), 785-793.

Lazarovici, D. \& Reichert, P. (2015). Typicality, Irreversibility and the Status of Macroscopic Laws. Erkenntnis 80 (4), 689-716.

Lebowitz, J. (1993). Macroscopic laws, microscopic dynamics, time's arrow and Boltzmann's entropy. Physica A: Statistical Mechanics and its Applications 194 (1-4), 1-27.

Malament, D. \& Zabell, S. (1980). Why Gibbs phase averages work. The role of ergodic theory. Philosophy of Science 47 (3), 339-349.

McCoy, C. D. (2020). An Alternative Interpretation of Statistical Mechanics. Erkenntnis 85 (1), 1-21.

Myrvold, W. (2016). Probabilities in Statistical Mechanics. In Hitchcock, C. \& H'Ajek, A. (eds.), The Oxford Handbook of Probability and Philosophy (pp. 573-600) Oxford: Oxford University Press.

Robertson, K. (2020). Asymmetry, Abstraction, and Autonomy: Justifying Coarse-Graining in Statistical Mechanics. British Journal for the Philosophy of Science 71 (2), 547-579.

Shenker, O. (2020). Information vs. entropy vs. probability. European Journal for Philosophy of Science 10 (1), 1-25.

Sklar, L. (1993). Physics and Chance: Philosophical Issues in the Foundations of Statistical Mechanics, Cambridge: Cambridge University Press.

Uffink, J. (2007). Compendium of the foundations of classical statistical physics. In J. Butterfield, \& J. Earman, (Eds.), Handbook for philosophy of physics. Amsterdam: Elsevier. Available online at http://philsci-archive.pitt.edu/archive/00002691/

Wallace, D. (2011). The logic of the past hypothesis. In Barry Loewer, Eric Winsberg \& Brad Weslake (eds.), Currently-unnamed volume discussing David Albert's "Time and Chance". Available online at http://philsci-archive.pitt.edu/8894/

Wallace, D. (2020). The necessity of Gibbsian statistical mechanics. In Allori, V., (Eds.), Statistical Mechanics and Scientific Explanation Determinism, Indeterminism and Laws of Nature-World Scientific. (pp. 583-616.) London: World Scientific.

Wilhelm, I. (2019). Typical: A Theory of Typicality and Typicality Explanation. British Journal for the Philosophy of Science. 1-23.

JAVIER ANTA is a graduate student in the Department of Philosophy at the University of Barcelona and a Student Member at LOGOS and BIAP. He received his B.A. in Philosophy from the University of Seville. His research interests include the philosophy and history of physics and the philosophy of information.

Address: Universitat of Barcelona, Departament de Filosofia, Carrer de Montalegre 6-8, $4 .{ }^{\circ}$ floor (Office 4013), PC: 08001 Barcelona. Email: antajav@gmail.com / anta@ub.edu

ORCID: 0000-0002-4409-7719 